\title{
Chris Gilleard and Paul Higgs (2013). Ageing, Corporeality and Embodiment. London \& New York: Anthem Press, 198 pp. ISBN 9780 857283290 (hardback)
}

\author{
REVIEWED bY JESSICA FINLAY*
}

In gerontology and broader academic circles, the ageing body remains largely under-theorized. Ageing, Corporeality and Embodiment addresses this critical lacuna in the literature by proposing an intervention into how we think about the ageing body and understand embodied everyday experiences of later life. Chris Gilleard and Paul Higgs craft a nuanced argument drawing upon the corporeality of later life as well as embodiment of the social. Two overarching concepts structure their discussion: embodied identities (e.g. gender, race, disability and sexuality) and embodied practices (e.g. sex, cosmetics/fashion, fitness/exercise and aspirational medicine). Gilleard and Higgs frame the ageing body as a site of embodied and contested difference, which generates alternative understandings of how the body is performed, challenged and negotiated. This book represents a longoverdue step advancing our knowledge of the complex and multifaceted relationship between the body and ageing.

Ageing, Corporeality and Embodiment draws on historical impacts of the 1960s: a key period that cultivated advances in the sociology of the body. The rise of counter-cultures and politics of representation in this time period 
International Journal of Ageing and Later Life

motivated new forms of self-care and self-representation that led to diverse experiences and expectations of ageing. Gilleard and Higgs portray the resulting "new ageing" as moving beyond traditional notions of "not becoming old" to "becoming old" differently. Many of the struggles to generate different identities and practices of ageing revolve around the body. The body represents a critical axis of orientation offering both possibility and constraint. The ageing body is a key site where traditional fears of old age mix with new hopes: "for ageing differently, for not having to become old on other people's terms" (p. 29).

Older people are gendered, raced, disabled and sexed in unique ways. Chapters following the introduction explore embodied identities of older adults: "identities and lifestyles based around some collectively shared set of bodily distinctions: distinctions of how particular bodies act, look or both" (p. ix). Gilleard and Higgs connect the embodiment of ageing to the 1960s sexual revolution, which rejected biological determinism of the sexed body. This led to questioning of divided understandings of bodies as corporeal units versus social identities. The authors explore this through the gendered nature of ageing, as men and women become embodied actors engaging in gendered performances (e.g. anti-ageing consumerism, exercise and fitness, appearance and sexual practice). The 1960s privileging of youthfulness and consumerism are no longer ideals for younger generations; rather, they have become models for all regardless of age, gender, class and other markers of difference.

The authors further observe that the majority of gerontological research involves a de-gendered and de-racialized corporeal lens. Gender and race are predominantly considered supplemental categories of structural disadvantage (p. 52). The authors argue for creating alternative narratives of ageing that counter dominant white and male accounts of ageing. Through this alternative approach, the ageing body is a site to contest not only ageism, but racism and sexism as well.

The following chapter in Ageing, Corporeality and Embodiment tackles the embodied identities of disabled older people. Adults often become disabled in later life in unique ways not addressed by existing disability scholarship. Disability studies tend to marginalize issues of age and ageing by presuming that disabled people have a static identity. This chapter also addresses disability studies' historic turn to citizenship and civic rights 
Ageing, Corporeality and Embodiment. Book review

instead of subjective embodiments. Critical scholarship displaced the corporeality of bodily impairment in favour of the social model of disability which investigates how institutions and social structures disable citizens. This is complicated, because disability often involves very real corporeal experiences. Gilleard and Higgs attempt to bridge the social/corporeal divide in their integration of age and disability studies. They use the ageing body as a key site to consider what disability really means to older people, such as what experiences of disability they negotiate, and bodily and social struggles they face.

In the subsequent chapters on sex and sexuality (Chapters 6 and 7), the authors transition the discussion to embodied practices. They describe these as "practices of self-care and self-expression that are mediated by society in, and through, the autonomous body" (p. ix). Practices include sex; cosmetics, clothing and fashion; fitness and exercise; and aspirational medicine. The chapter on fitness and exercise includes an intriguing critical discussion on the associations between physical activity and virtuous public citizenship. Health and fitness are virtues that citizens are expected to cultivate at all ages, and the "new ageing" ethic embedded within successful and productive ageing paradigms includes the pursuit of fitness. The authors provide examples of older people's individual efforts to integrate exercise, active leisure and consumption in later life. They allude to underlying neoliberal models of health policy, in which citizens engage in active ageing practices to maintain virtuous citizenship under Foucauldian governmentality.

In summary, Ageing, Corporeality and Embodiment represents a critical intervention in ageing scholarship. It contests traditional notions of the ageing body and considers how the body represents a mediating force between individuals and society. Gilleard and Higgs challenge how the body is "read" and offer alternative frameworks for how people "become old" differently. The contestations over alternative embodiments in this book focus on discourses, practices and identities of the third age. It is important to note that the fourth age, generally demarcated by experiences of illness and frailty in the final stage of life, is absent from this book. As the authors acknowledge, addressing themes of the fourth age would involve a different perspective and project altogether. Yet the experiences of the oldest old are inescapably intertwined with embodied identities and 
International Journal of Ageing and Later Life

practices of later life, and further complicate the relationship between the body and society. I look forward to ensuing discussions of alternative ageing embodiments that this book will stimulate. Gilleard and Higgs continue to transform how we understand later life, and this perceptive book generates compelling queries that need investigating. 